\title{
Morphological peculiarities of the trigeminal nerve roots and ganglia in human fetus
}

Discipline of anatomy, Department I - preclinical disciplines, Faculty of medicine, University "Ovidius" Constanţa

\begin{abstract}
The study of the intracranial traject of the trigeminal nerve was performed through a dissection of 40 human fetuses whose ages ranged between 4 and 9 months. We examined: the apparent origin of the nerve, the traject, stating the length and width of the roots and of the triangular plexus, the antero-posterior width of the trigeminal ganglion and its transverse diameter, measured between the two extremities (horns). The anatomical landmarks were considered left versus right for each fetus and the trigeminal ganglia were studied only in terms of macroscopy: form and relations. We found that the sensory root length increases greatly between the fourth and the fifth month (more than 1 $\mathrm{mm}$ ), from six to seven months the root length present a stagnation and in the eighth month its increase in length is very significant, about $4 \mathrm{~mm}$, while in the ninth month we also recorded an increase of about $2 \mathrm{~mm}$. The motor root may have two separate fiber bundles, both as cylinders, which can be present from the apparent origin and up to the mandibular nerve; near their origin of the two bundles are separated by nervous tissue. The trigeminal ganglia has, most often, a semilunar aspect, thus justifying its name. Other times it may be irregular, triangular or quadrilateral (rectangular). There are situations when the trigeminal ganglion shows three lobes, each corresponding to one of its three terminal branches.
\end{abstract}

Keywords: ganglia, trigeminal nerve, fetus

\section{Iliescu D.M.}

Department of Anatomy, Faculty of medicine, University "Ovidius" Constanţa, Romania

Aleea Universitatii, Nr. 1, Campus B

Constanţa, Romania

dan@anatomie.ro

\section{Introduction}

The trigeminal nerve is the nerve of the first branchial arch (mandibular arch). The trigeminal ganglia results from the merge of two buds, ophthalmic and maxillo-mandibular, which are separated in inferior vertebrates. The motor root, branhio-motor $[1,2]$ is aimed towards the mesenchyme of the first arch, differentiated into the masticatory apparatus. In stage 11 (approximately 24 days), within the neural tube occurs a condensation of the neural crest corresponding to the trigeminal ganglion, on the side of rombencephalon. In stage 12 (about 26 days), the fourth cerebral ventricle appeared and the trigeminal ganglion is easily distinguished; in stage 13 (about 28 days), the cranial nerves are present; in stage 14 (about 32 days) the trigeminal ganglion is voluminous; in stage 15 (about 33 days) are present all nuclei of the trigeminal nerve; in stage 18 (about 44 days) the pons and the cerebellum are rudimentary; at the end of the embryonic period (stage 23), are developed all elements of the nervous system, the nerves and the sense organs presenting their final appearance.

At the level of the apparent origin, the of sensory and motor roots are sometimes separated by a bridge of nervous tissue, lingula of Wrisberg [1,3]. The sensory root, after its exit from the neuraxis, gradually flattens in vertical direction, showing two faces, one superior and one inferior, with a width of $4 \mathrm{~mm}$ out of the pons, $5-6 \mathrm{~mm}$ in the middle portion and 7-8 $\mathrm{mm}$ to the upper border of the petrous part. It consists of 40-60 nerve bundles, not only close one 
to another, but also linked by numerous anastomoses [3].

The motor root, $1.5-2 \mathrm{~mm}$ wide, passes on the internal border of the sensitive one to the level of Meckel's cavum. Upon entering the cavum, the root undergoes a torsional motion on its axis, its medial bundles becoming lateral and vice versa. At the same time it is flattened from top to bottom and gradually increasing in width to the end of it. The motor root does not connect to the sensorial but stay close to it [3]. The root is formed by 5-7 nerve bundles, no more than 10 [3]. The motor root is placed successively above, medially and then below the sensitive root, and then laterally in relation to the trigeminal ganglion. The trigeminal ganglion is located in a splitting of the dura mater within the middle cranial fossa, the Meckel's cavum, on the anterior superior face of the petrous part of the temporal bone $[1,3,4]$.

On the upper border of the petrous part, the trigeminal nerve impose the trigeminal notch (of Gruber); over the nerve pass the tent of the cerebellum and the superior petrous venous sinus $[1,3]$.

The trigeminal ganglion has a semilunar shape (or beans shape), strongly flattened, and with the hilum directed postero-superior and the convex border anterior-inferior. It has two faces: the anterolateral, relative to dura mater, which adheres intimately, making it difficult dissection and the postero- medial, corresponding to the bone, which is separated by a fibrous lamina from the dura mater. The anterior face intimately adheres to the fibrous sheath while the posterior is connected by loose connective tissue [3].

\section{Material and method}

The study of the intracranial traject of the trigeminal nerve was conducted through the dissection of 40 human fetuses whose ages ranged between 4 and 9 months. We work on 3 fetuses of 4.5 months, 2 fetuses of 5 months, 3 fetuses of 5.5 months, 8 fetuses of 6 months, 6 fetuses of 6.5 month, 9 fetuses of 7 month, 5 fetuses of 8 months and 2 fetuses of 9 months. After a formalization that took about a month, the calvaria was removed, the meninges sectioned and the encephalic mass of the cerebral hemispheres removed, without injuring the brainstem. After these operations the fetuses were placed back in formalin solution for 2-3 days, as long as in many cases the brain was still soft and does not allow maneuvers on the trigeminal nerve, most commonly the damage occurring in the encephalic intracranial part. Then followed the trigeminal nerve root dissection from the apparent origin on the anterolateral aspect of the pons to the top of the petrous part of the temporal, aiming in particular the relations that they establish with other cranial nerves. Then we carefully examined the trigeminal notch, evaluating its depth and the relation with the apex of the bone, and the existence or not of a root canal, formed by the meninges or by bone. The dissection of the triangular plexus and of the trigeminal ganglion followed, a rather difficult dissection due to adherence of these structures to Meckel's cavum. All of these elements were measured, evaluating the length and width of the root and triangular plexus, the anterior-posterior width of the trigeminal ganglion, and its transverse diameter, measured between the two extremities (horns). The anatomical landmarks were observed left versus right for each fetus and photographed separately and together. We did not perform sections or histological studies, nor were counted the nerve fibers in the roots or branches of the trigeminal nerve. Also, the trigeminal ganglia have been studied only in macroscopic point of view: the shape, dimensions, and relations.

\section{Results}

The trigeminal nerve apparent origin is on the anterior side of the pons at about midway between its upper and lower borders. In general, it remains away from the basilar artery and its main branches. From our findings it seems that the apparent origin of the trigeminal nerve, most often, is closer to the apparent 
origin of the facial, intermediate and vestibulocohlear nerves and further to the apparent origin of the abducens nerve. More rarely, the distance between the origins of the VII, VIIbis and VIII cranial nerves and the trigeminal nerve is smaller, their dural nerve sheaths being connected to one another. At its apparent origin, the trigeminal nerve, most commonly, is flattened in cranio-caudal direction and rarely takes the shape of a cylindrical cord (rounded). The direction of the trigeminal nerve roots, from the origin to the top of the petrous part of the temporal, is often oblique antero-inferior, rarely transverse postero-anterior and seldom oblique antero-superior.

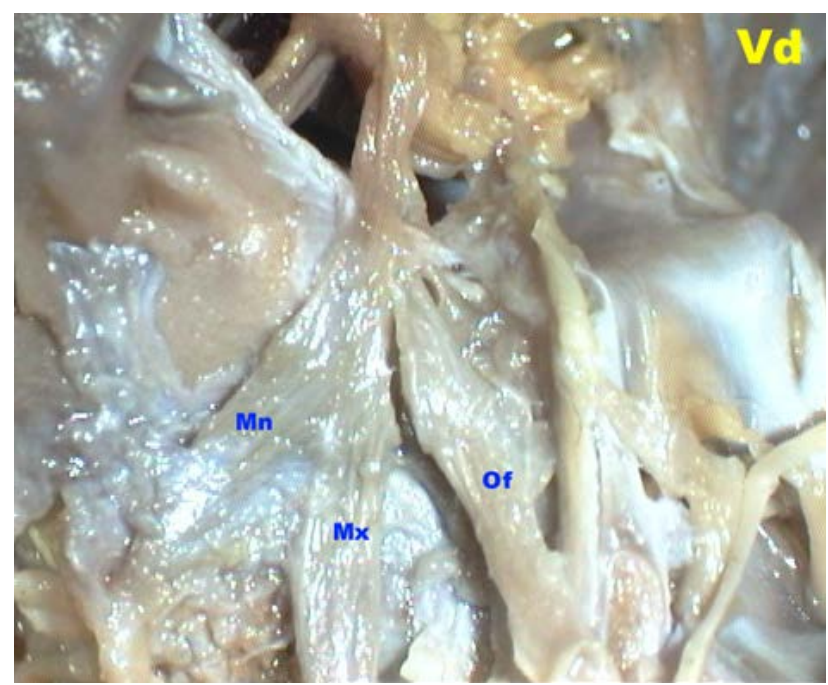

Figure 1 - Thick motor root, with a caliber of a half of the sensorial one, formed by 5 bundles; at origin is located infero-lateral to the sensorial one, then passes laterally and, from the upper border of the petrous part, passes inferiorly

The sensory root of the trigeminal nerve has a relatively constant length to mid-sixth month of the intrauterine life, as its dimensions increase rapidly in the seventh month, especially in eight to nine months. In the fourth month we found that the sensory root length ranged from 8.4 to $9.8 \mathrm{~mm}$, with no differences in length right to left. We found the root width between 1.8-2.2 mm, with some minor differences right to left of 0.1-0.2 mm, without establishing dominance in one direction or another. In the fifth month the root length ranged from $10.5-11 \mathrm{~mm}$, with differences of $0.2-0.3$ $\mathrm{mm}$ on one side or another. The sensory root width was between 2-3.5 mm, 0.2-0.5 mm differences for root straight. In the sixth month length ranged from 9.5 to $11.5 \mathrm{~mm}$, with differences of $0.5-0.8 \mathrm{~mm}$ from one side or another, without being able to establish side dominance. We found a width of between 2-3 mm, with differences of 0-0.5 mm, one side or the other. In the seventh month the length of the roots ranged from 14.8 to $15 \mathrm{~mm}$, with differences of $0.2-0.5 \mathrm{~mm}$ and the width was 2.8 to $2.9 \mathrm{~mm}$, with differences of 0 to $0.1 \mathrm{~mm}$. In the eighth month the length ranged from 14.8 to $15 \mathrm{~mm}$, with differences of 0 to $0.2 \mathrm{~mm}$ and width of 4.5 to $4.8 \mathrm{~mm}$, with differences of $0-0.3 \mathrm{~mm}$. In the ninth month the root length was 16.5 to 16.8 $\mathrm{mm}$, with differences of $0-0.3 \mathrm{~mm}$ and $3.2-3.5 \mathrm{~mm}$ wide, with differences of $0-0.3 \mathrm{~mm}$.

At the level of the apparent origin of the sensory root may appear, frequently, two or three distinct thick bundles of nerve fibers that sometimes remain as such until the level of the trigeminal impression, thus bypassing the upper border of the petrous part. In these situations there is virtually no single trigeminal ganglion, undivided. In other cases, the bundles of the sensory root of the nerve remain as such only up to the upper border of the petrous part and then join one another to form the triangular plexus.

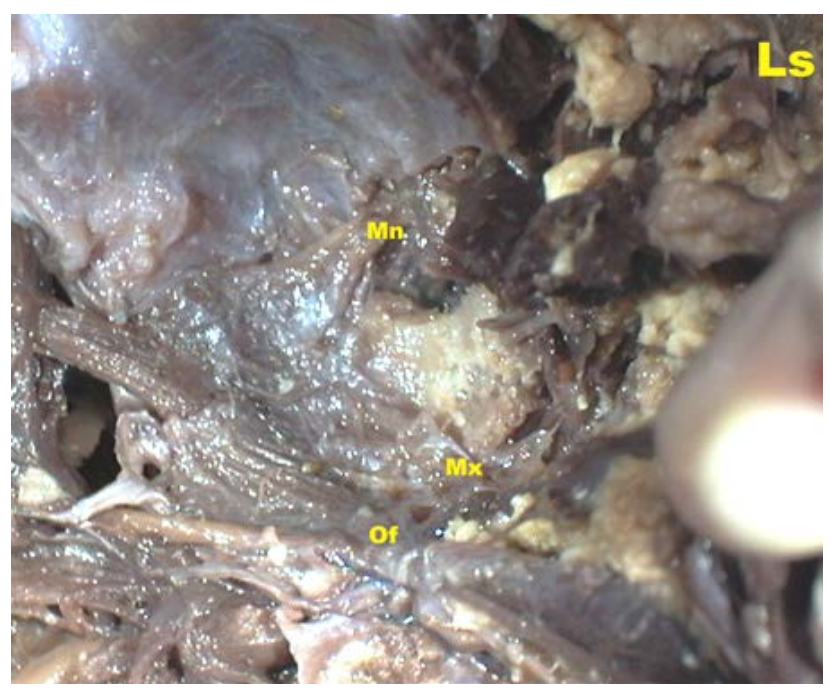

Figure 2 - Bilobate trigeminal ganglion, corresponding to the maxillary and mandibular nerves; the ophthalmic nerve starts from the upper part of the petrous part of the temporal bone. 
The motor root is clearly visible at the apparent origin of the trigeminal nerve, the most commonly being attached to the sensory root, and rarely can be separated from this, with its own sheath. Although frequently the motor root of the trigeminal nerve is located medial to the apparent origin and in the same plane as the sensory root, the motor root can sometimes be located supero-medial to the sensory root, pass on the top and then infero-medial to it. Sometimes the motor root can go together, in the same sheath, with a bundle from the sensitive root. The motor root may have two separate fiber bundles, both cylinders, which can be present from its apparent origin and up to the mandibular nerve; near the origin the two bundles are separated by a bridge of nervous tissue.

Most often, the motor root is placed on the inferior face of the triangular plexus, rarely posterior and very rare anterior to it.

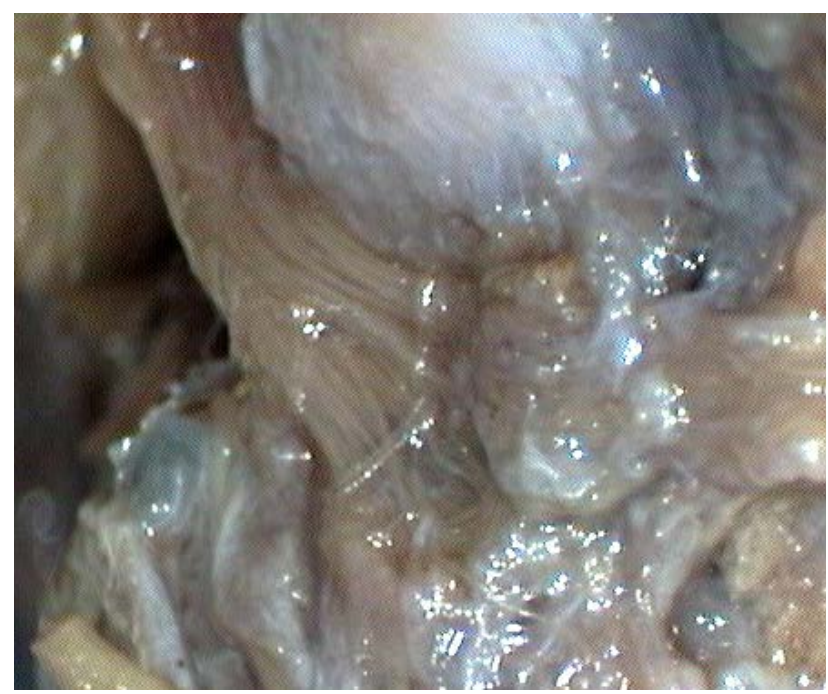

Figure 3 - Triangular plexus that starts posterior to the upper part of the petrous part of the temporal bone with parallel and crossed fibers. The bundles of the three nerves, prior to their entrance into the trigeminal ganglion.

At the upper border, over the trigeminal nerve roots is almost always a dural bridge that transforms the notch into a hole or channel. Sometimes the foramen demarcation may be marked by a thin bony lamella, incomplete (less complete), thus forming a bony foramen or bony canal.
The length and width of the triangular plexus are highly variable in relation with the fetal age. The plexus is short and narrow until the seventh month, after which greatly increases its width, but the length stay variable, most often being short. The plexus inclination is very high in small months and gets smaller after the sixth month.

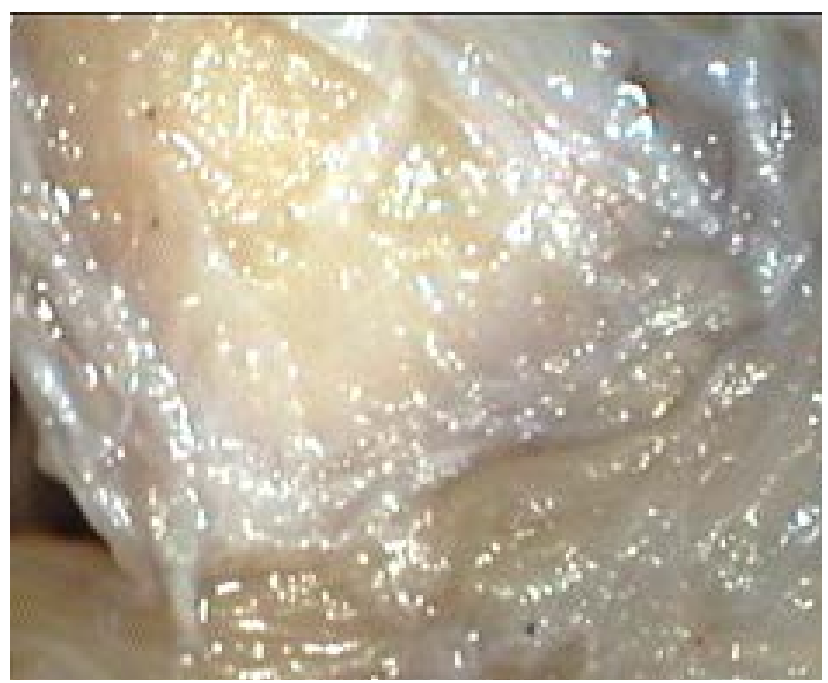

Figure 4-Incomplete osteo-dural bridge on the trigeminal notch; the formation of the triangular plexus starts posterior to the petrous part of the temporal bone

The plexus fibers are very irregularly arranged, intersecting in all directions and anastomoting, but simultaneously there are fibers parallel to each other.

There are situations when the fibers of the triangular plexus, irregularly distributed, pass only toward the maxillary and mandibular nerves and taking no part in the formation of the ophthalmic nerve.

In other cases we meets separated plexus fibers into two parts, the medial and the lateral, that enters the concavity of the trigeminal ganglion.

Regarding the trigeminal ganglion, we found the antero-posterior width of the middle portion (the widest), until the fifth month, from 2.2 to $3.2 \mathrm{~mm}$, with differences in left to right of 0,2 - $0.6 \mathrm{~mm}$. Only in one case the width was $4 \mathrm{~mm}$. In the fifth month the antero-posterior width was between 3-4 $\mathrm{mm}$, with differences of 0 to $0.2 \mathrm{~mm}$ in width. In the sixth month it may vary greatly, ranging from 2.8 to $3.8 \mathrm{~mm}$, with differences of 0.2 to $0.3 \mathrm{~mm}$, predominantly on the 
right. In the seventh month it was $4 \mathrm{~mm}$ on both sides. From the eighth month there is a large increase in the antero-posterior width of the ganglia, with 4.5 to 5.2 $\mathrm{mm}$, with small differences in right / left of 0.2-0.3 $\mathrm{mm}$.

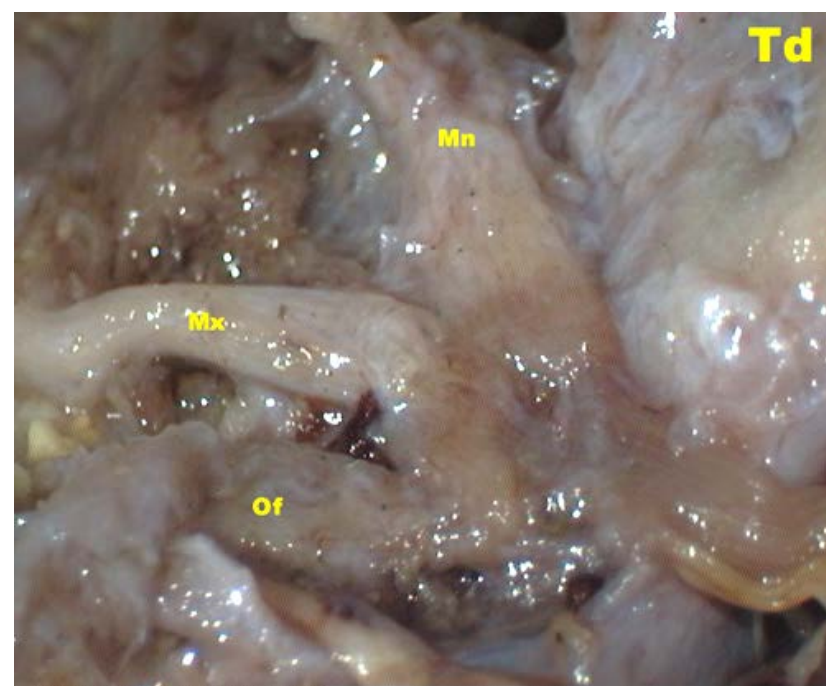

Figure 5 - Trilobate trigeminal ganglion. The triangular plexus is narrow, with a large motor root placed medially to the petrous part of the temporal bone.

The transverse width of the trigeminal ganglion, i.e. between its two horns, is between 5.5 to $6.8 \mathrm{~mm}$, until the fifth month, with $0-0.3 \mathrm{~mm}$ difference between the two parties. In the fifth month the width is 8 to $8.2 \mathrm{~mm}$, with differences of 0 to 0.2 $\mathrm{mm}$. In the sixth month the values are similar, being 7.5-8.5 mm, with differences right / left of 0-0.5 mm. In the seventh month the width is 8.5 to $9.8 \mathrm{~mm}$ and with the differences of 0 to $0.2 \mathrm{~mm}$ between the two parties. After eight months there has been a marked increase in the trigeminal ganglion width between its two horns, being of $12.5-15 \mathrm{~mm}$, with differences in right / left of 0.4-0.5 mm.

The trigeminal ganglia is most often semilunar in shape, thus justifying its name. Other times it may be irregular, triangular or quadrilateral (rectangular).

There are situations when there is a three lobes trigeminal ganglion, each corresponding to one of its three terminal branches. In other cases there is only the lobulation of the ophthalmic or mandibular nerve.

From the convexity or the anterior border of the trigeminal ganglion leaves the terminal branches, more or less spaced apart, often resulting in sharp angles. Very often, at origin, the maxillary nerve is close to the ophthalmic nerve or mandibular nerve. More rarely, the three branches are located in the same plane, the most common being located in different planes: either ophthalmic nerve is the most superficial (most common situation) or mandibular nerve is most superficial.

In some cases from the anterior part of the ganglion start two bundles: one lateral (the mandibular nerve) and another medial, which will give birth to the ophthalmic and maxillary nerves.

Sometimes from the front of the trigeminal ganglion detach only maxillary and mandibular nerves, while the ophthalmic may be individualized posterior to the ganglion and, not in a few cases, even on the top of the petrous part.

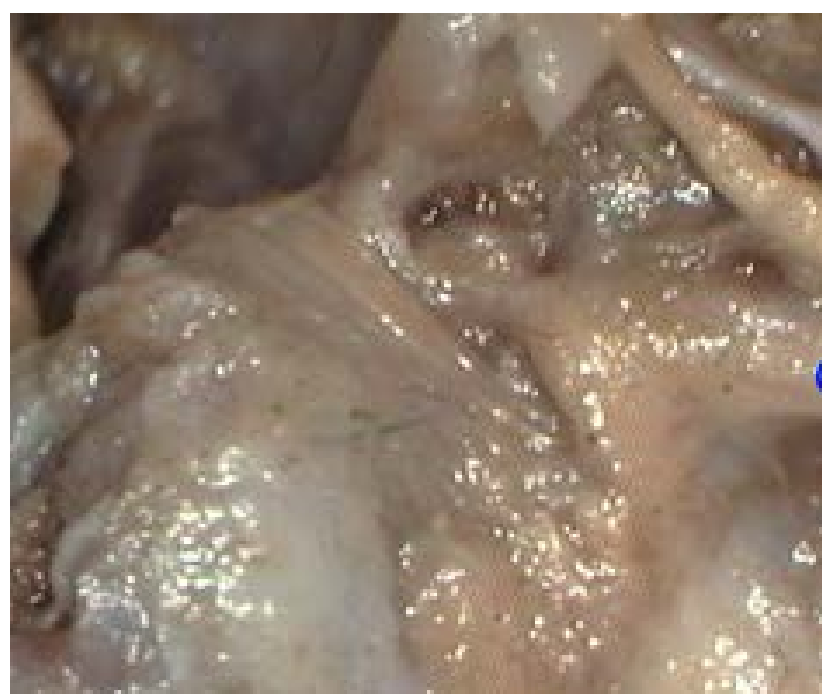

Figure 6-Small triangular plexus, slightly inclined and typical trigeminal ganglion; the fibers of the ophthalmic nerve start next to the upper margin of the petrous part of the temporal bone.

Very rarely this individualization can appear right from the root sensory, posterior to the upper border of the petrous part, yet presenting at this level its own sheath that separates it from the rest of the nerve fibers. 


\section{Discussion}

According to [2] the somato-sensitive and the viscero-sensitive nerve centers of the trigeminal nerve are on the alar lamina and in the dorso-lateral part of the fundamental lamina is a motor column that innervate the muscles originating in the branchial arches, called the branhio-motor column, which exists only in brainstem and include the neuromotor nuclei of striated branchial muscles (IX), facial muscles (VII) and the muscles of mastication (V). We found that the sensory root of the trigeminal nerve is rarely cylindrical at its apparent origin, being flattened cranio-caudally. According to [3], the sensory root, after its exit from the neuraxis, is gradually flattened in a vertical direction, a process which continues up to the upper edge of the temporal petrous part.

Following our results, we found that the length of the sensory root increases greatly between the fourth and fifth month (more than $1 \mathrm{~mm}$ ), between six to seven months the root length present a stagnation, and starting the eighth month its increase in length is very large, about $4 \mathrm{~mm}$, in the ninth month also we recorded an increase of about $2 \mathrm{~mm}$. Significant length difference between the two sides, right / left, we met only the sixth and seventh months (0.5-0.8 $\mathrm{mm}$ ); in other months these differences were very small (months five, eight and nine) or even nonexistent, as the right and left roots were same length (in particular in the fourth, but also, in some cases, fifth, eight and nine months).

The transverse width of the sensory root of the trigeminal nerve suffers an increase between the fourth and the fifth month of $1-1.3 \mathrm{~mm}$, remaining relatively constant until the seventh month, from the eighth month also shows a significant increase of approximately $1-1.3 \mathrm{~mm}$. In all months there is a difference of 0.2-0.5 mm between the right and left, but only in the fifth month this difference is more common on the right.

The motor root of the trigeminal nerve is cylindrical (rounded) at its apparent origin, rarely being slightly flattened cranio-caudally. [3] states that the motor root passes on the internal border of the sensitive one up to the Meckel's cavum level. Upon entering the cavum, the root undergoes a torsional motion on its axis. At the same time it is flattened top down and gradually increases in width until its end. According to [3], the motor root does not enter into connection with the ganglion but stay around it; sometimes the motor roots may be included in the dural sheath of the inferior face of the trigeminal ganglion.

[1] states that the trigeminal ganglia results from the merger of two buds, ophthalmic and maxillo-mandibular, which are separated in from lower vertebrates, which explains the possibility of two or three-lobed ganglion. According to [5], this was due to the fact that the development of nerve cell and axons three were not done at the same time, but from time to time; for example the maxillary appears which the step of 30 somites while the mandibular occur within somite stage 32 .

According to [3], the trigeminal ganglion has a semilunar or beans shape, strongly flattened, with the hilum facing convex posterior superior and anteriorinferior; we found a variable shape from one case to another.

The trigeminal ganglion increases its anteroposteriorly width with $0.8 \mathrm{~mm}$ between the fourth and fifth months, while its size remains constant in sixth and seventh months. From the eighth month there is a marked increase in the width of 1-1.2 mm. Note the great variability of the antero-posterior width within the sixth month, when between a fetus and another we encountered differences of up to one $\mathrm{mm}$. The transverse width (between the two horns) increases $1.5-2 \mathrm{~mm}$ between the fourth and fifth month, after which it remains constant until the seventh month, when there was again an increase in transverse width ganglion of 0.3 to $1.6 \mathrm{~mm}$, and this increase is marked in the eighth month, the node increases from 2.7 to $5.2 \mathrm{~mm}$. The differences between right and left widths are greater in the sixth month (up to 0.5 $\mathrm{mm}$ ) and eight to nine months $(0.4-0.5 \mathrm{~mm})$. In other months the differences are smaller, being $0-0.3 \mathrm{~mm}$. According to [6] the number of embryonic cells in the trigeminal ganglion does not change significantly with gestational age, but the cell diameter, area and perimeter have increased significantly. 
From the traject of the trigeminal nerve, between the origin and the upper edge of the petrous part of the temporal, it worth mentioning the anastomoses that we have isolated between the root of the trigeminal nerve and the facial nerve, anastomosis which may be autonomic fibers of the facial which would continue their traject through the trigeminal nerve.

Even if, within the nerve traject, we met no anastomosis between the motor and sensory roots, in situations when the motor root crosses the triangular plexus, it may also receive some sensory fibers. We believe that this could happen up to the apparent origin of the nerve, which would explain the partial anesthesia installed after the neurotomy of the sensory root, demonstrated since 1956 by Sicard [quoted by 1]. The electron microscope proved that, in fetuses aged 20 weeks, part of the trigeminal nerve fibers showed morphological characteristics of mature nerve fibers; they are organized in a medio-lateral direction reflecting the medio-lateral sequence of the three primary nerve branches.

For most authors, the trigeminal ganglion is contained in a duplication of the dura mater, which is extended on the three terminal branches $[1,4,7]$, but [8], on serial histological sections, found that dura and the arachnoid stop at the level of the trigeminal ganglia and do not extend over the three branches of the trigeminal nerve, which is embedded in special, different sheaths.

Most often the trigeminal ganglion justifies its name, being semilunar, but it may have other shapes: rectangular, with large axis oriented transversally, irregular or can not be classified in any geometric shape, but the most interesting aspect is the lobate trigeminal ganglion: two or three lobes, the latter aspect looking like a clover leaf, each leaflet of the node leaving one branch of the trigeminal nerve. The leaflets are well separated from each other, and may appear from the level of the triangular plexus.
1. Fontaine C. \& Chevrel J.P. (1995). Anatomie clinique. Tête et cou. Ed. Springer - Verlag, Paris, pp. 137-140

2. Drews U. (1994). Atlas de poche d'Embriologie, Ed. Flamarion, Paris, pp. 226.

3. Testut L. (1922). Traité d'Anatomie Humaine. Tome troisième. Système nerveux périphérique. Organes des sens. Appareil de la respiration et de la phonation. Ed. Gaston Doin, Paris, pp. 5559

4. Kamina P. (1996). Tête et cou. Nerfs crâniens et organes des sens. Tome 2. Ed. Maloine, Paris, pp. $42-43$

5. Scott L. \& Atkinson M. (1999). Compartmentalization of the developing trigeminal ganglion into maxillary and mandibular divisions does not depend on target contact. J Anat. 195(1), 137-145

6. Wu L., Zhang H., Liao L., Dadihan T., Wang X. \& Kerem G. (2013). Trigeminal ganglion morphology in human fetus. Microsc. Res. Tech.,

7. Rouvière H. (1981). Anatomie humaine. Tome 1. Tete et cou. Paris

8. Kehrli P., Maillot C. \& Wolff M.J. (1997). Anatomy and embryology of the trigeminal nerve and its branches in the parasellar area.

Neurol Res. 19(1), 57-65 